\title{
THE STOCHASTIC VOLTERRA EQUATION
}

\author{
Bernt Øksendal and Tu-Sheng Zhang \\ Department of Mathematics \\ University of Oslo \\ Box 1053 Blindern \\ N-0316 Oslo, NORWAY
}

\section{Abstract}

We study the stochastic (Skorohod) integral equation of the Volterra type

$$
X_{t}(\omega)=Y_{t}(\omega)+\int_{0}^{t} b(t, s) X_{s}(\omega) d s+\int_{0}^{t} \sigma(t, s) X_{s}(\omega) \delta B_{s}(\omega)
$$

where $Y, b$ and $\sigma$ are given functions; $b$ and $\sigma$ are bounded, deterministic and $Y_{t}$ is stochastic, not necessarily adapted. The stochastic integral $(\delta B)$ is taken in the Skorohod sense.

In general there need not exist a classical stochastic process $X_{t}(\omega)$ satisfying this equation. However, we show that a unique solution exists in the following extended senses:

(I) As a functional process

(II) As a generalized white noise functional (Hida distribution).

Moreover, in both cases we find explicit solution formulas. The formulas are similar to the formulas in the deterministic case $(\sigma \equiv 0)$, but with Wick products in stead of ordinary (pointwise) products. 



\title{
THE STOCHASTIC VOLTERRA EQUATION
}

\author{
Bernt $\varnothing \mathrm{ksendal}$ and Tu-Sheng Zhang \\ Department of Mathematics \\ University of Oslo \\ Box 1053 Blindern \\ N-0316 Oslo, NORWAY
}

\section{$\S 1$. Introduction}

The classical (deterministic) Volterra equation of the second kind has the form

$$
X_{t}=Y_{t}+\int_{0}^{t} \gamma(t, s) X_{s} d s \quad 0 \leq t \leq T
$$

where $Y_{t}, \gamma(t, s)$ are given functions, $T>0$ is a given constant. This equation occurs in many applications, some of which are described in [GLS]. See also [T] and Example 1.1 below. Suppose now that the system is randomly perturbed or that there is insufficient/noisy information about the function $\gamma(t, s)$. In both cases a possible mathematical formulation would be to put

$$
\gamma(t, s)=b(t, s)+\sigma(t, s) \cdot W_{s},
$$

where $b(t, s)$ and $\sigma(t, s)$ are deterministic functions and $W_{s}=W_{s}(\omega) ; \omega \in \Omega$ (a probability space) denotes "white noise" (see definitions below). We also allow $Y_{t}=Y_{t}(\omega)$ to be random. This gives - formally - the equation

$$
X_{t}(\omega)=Y_{t}(\omega)+\int_{0}^{t} b(t, s) X_{s}(\omega) d s+" \int_{0}^{t} \sigma(t, s) X_{s}(\omega) W_{s}(\omega) d s^{\prime \prime}
$$

where the last term (in quotation marks) remains to be defined.

If $Y_{t}$ is an adapted stochastic process, then it is natural to assume that a solution $X_{t}$ of (1.3) must be adapted too, and this leads to the following interpretation of (1.3):

$$
X_{t}(\omega)=Y_{t}(\omega)+\int_{0}^{t} b(t, s) X_{s}(\omega) d s+\int_{0}^{t} \sigma(t, s) X_{s}(\omega) d B_{s}(\omega)
$$

where the last term denotes the usual Ito integral and $B_{t}(\omega)$ denotes Brownian motion whose t-derivative is $W_{t}(\omega)$ (in distribution sense).

In this paper we are mainly interested in the case when $Y_{t}$ is not adapted. In this case we of course cannot expect $X_{t}$ to be adapted and then the Ito integral in (1.4) is not 
defined. However, equation still makes sense if we replace the Ito integral by the more general Skorohod integral:

$$
X_{t}(\omega)=Y_{t}(\omega)+\int_{0}^{t} b(t, s) X_{s}(\omega) d s+\int_{0}^{t} \sigma(t, s) X_{s}(\omega) \delta B_{s}(\omega)
$$

REMARK. The Skorohod integral

$$
\int_{0}^{T} Z_{s}(\omega) \delta B_{s}(\omega)
$$

is defined for all processes $Z_{t}(\omega)$ (adapted or not) such that

$$
\int_{0}^{T} E\left[Z_{s}^{2}\right] d s+\sum_{m=1}^{\infty}(m+1) !\left\|\tilde{f}_{m}\right\|^{2}<\infty
$$

Here $\tilde{f}_{m}\left(t_{1}, \cdots, t_{m}, t\right)$ is the symmetrization of $f_{t}^{(m)}\left(t_{1}, \cdots, t_{m}\right)$, where $f_{t}^{(m)}$ is the m'th order term in the Wiener-Ito chaos expansion of $Z_{t}$ :

$$
Z_{t}(\omega)=\sum_{m=0}^{\infty} \int_{\mathbf{R}^{m}} f_{t}^{(m)}\left(t_{1}, \cdots, t_{m}\right) d B_{t_{1}} \cdots d B_{t_{m}}
$$

If $Z_{s}(\omega)$ is adapted, then the Skorohod integral coincides with the Ito integral [NZ].

We will use (1.5) as our mathematical model for a randomly perturbed Volterra integral equation, or a stochastic Volterra integral equation for short. The purpose of this paper is to study the existence and uniqueness of a solution of (1.5). Moreover, we will find an explicit solution formula. It turns out that in general (without strong conditions on $Y_{t}, b$ and $\sigma$ ) there does not exist a (classical) stochastic process $X_{t}$ satisfying (1.5). However, we will prove that a solution exists (and is unique) in the following extended senses:

(I) As a functional process (see §3)

(II) As a generalized white noise functional (or Hida distribution) (see §4).

Skorohod Volterra equations with anticipating kernel (but non-anticipating initial condition $\left.X_{0} \equiv Y_{t}(\forall t)\right)$ have been studied in [PP], see also [BM] and the survey in [Pa]. In [O] the stochastic Volterra equation is studied in the setting of Ogawa-type integrals. To the best of our knowledge our paper is the first to discuss the Skorohod interpretation with anticipating initial conditions.

We now explain these two approaches in more detail:

(I) The functional process approach. ([LØU1],[LØU3],[HLØUZ]) (see §3 for details).

Here we regard the solution $X$ as a generalized stochastic process of the form

$$
X=X_{t}^{\phi}=X(\phi, t, \omega)
$$


where $\phi \in \mathcal{S}$ (the Schwartz space of rapidly decreasing functions on $\mathbf{R}$ ). Heuristically $X(\phi, t, \omega)$ can be regarded as the result of measuring $X$ (at time $t$ and in the experiment $\omega)$ through the averaging/test function or "window" $\phi$.

White noise $W$ may be regarded as such a functional process by the definition

$$
W(\phi, t, \omega):=W_{\phi_{t}}(\omega):=\int \phi_{t}(s) d B_{s}
$$

where $\phi_{t}(s)=\phi(s-t)$ is the window $\phi$ shifted by the amount $t$. Note that for each fixed $\phi$ both $X(\phi, \cdot, \cdot)$ and $W(\phi, \cdot, \cdot)$ are continuous stochastic processes. There is a striking formula for the Skorohod integral in terms of the Wick product $\diamond$ as follows (see Lemma 2.1):

$$
\int_{\mathbf{R}}(\phi * Y)_{t} \delta B_{t}=\int_{\mathbf{R}} Y_{t} \diamond W_{\phi_{t}} d t \quad \forall \phi \in \mathcal{S}
$$

where $*$ denotes convolution with respect to $t$, i.e.

$$
(\phi * Y)_{t}(\omega)=\int_{\mathbf{R}} \phi(t-s) Y_{s}(\omega) d s
$$

In view of this we say that a functional process $X_{t}=X_{t}^{\phi}(\omega)=X(\phi, t, \omega)$ is a solution of (1.5) if for all $\phi \in \mathcal{S}$ there exists a stochastic process $X_{t}=X_{t}^{\phi}$ such that

$$
X_{t}^{\phi}=Y_{t}^{\phi}+\int_{0}^{t} b(t, s) X_{s}^{\phi} d s+\int_{0}^{t} \sigma(t, s) X_{s}^{\phi} \diamond W_{\phi} d s ; 0 \leq t \leq T
$$

where we allow $Y_{t}=Y_{t}^{\phi}$ to be a functional process too. In $\S 3$ we show that a functional process solution of (1.5) exists under certain conditions on $Y_{t}^{\phi}, b(t, s)$ and $\sigma(t, s)$. Moreover, we give an explicit solution formula:

$$
X_{t}=Y_{t}+\int_{0}^{t} H(t, s) \diamond Y_{s} d s
$$

where $H(t, s)=H(t, s, \omega)$ is a random kernel constructed from $K(t, s, \omega):=b(t, s)+$ $\sigma(t, s) W_{\phi_{\rho}}(\omega)$.

(II) The generalized white noise functional (Hida distribution) approach [HKPS] (see §4)

In this setting we regard $X_{t}$ and the other elements of equations (1.5) as elements of the space $(\mathcal{S})^{*}$ of Hida distributions (or generalized white noise functionals). The pointwise white noise $W_{t}$ may be regarded as an element of $(\mathcal{S})^{*}$. By Corollary 3.4 in [L $\varnothing \mathrm{U} 2$ ] we have

$$
\int_{\mathbf{R}} Z_{t} \delta B_{t}=\int_{\mathbf{R}} Z_{t} \diamond W_{t} d t
$$


for all stochastic processes satisfying (1.6) (adapted or not). In view of this the natural interpretation of equation (1.5) in the Hida distribution setting is

$$
X_{t}=Y_{t}+\int_{0}^{t} b(t, s) X_{s} d s+\int_{0}^{t} \sigma(t, s) X_{s} \diamond W_{s} d s
$$

where $Y_{t}$ now is regarded as an element of $(\mathcal{S})^{*}$.

Note that by (1.13) the relation between the pointwise white noise $W_{t}$ and the functional process $W_{\psi}$ is (choose $Z_{t}=\psi(t)$ in (1.13)):

$$
W(\psi, 0, \omega)=\int_{\mathbf{R}} \psi(t) W_{t} d t
$$

i.e. $W(\psi)$ is the result of "smearing out" the singular noise $W_{t}$ by the test function $\psi$.

In this $(\mathcal{S})^{*}$-setting we prove an existence and uniqueness result for (1.14) and, here too, we obtain a solution formula of the type (1.12). Moreover, we show that the solution of (1.14) is actually in $L^{2}(\mu)$ under some conditions.

EXAMPLE 1.1 A number of applications of Volterra equations can be found in [GLS, p. 4-13]. Here we present an economic example, with a structure related to the population dynamics example presented in Ex. 2.2 in [GLS]. Our example leads to a stochastic Volterra equation of the form considered in this paper:

An investment in an economic production, for example the purchase of new production equipment, will usually have effects over a long period of time. Let $X(t, u)$ denote the capital distribution at time $t$ resulting from the investments which have age $u$ (i.e. which were made $u$ units of time ago). More precisely, let

$$
\int_{U} X(t, u) d u \text { denote the total capital gained }
$$

at time $t$ from all investments with age $u \in U$. Assume that

$$
\frac{\partial X(t, u)}{\partial t}+\frac{\partial X(t, u)}{\partial u}=-m(u) X(t, u)
$$

where $m(u) \geq 0$ denotes the age-dependent "death" rate of the equipmentment/machines involved in the production. Moreover, assume that the amount of new capital $X(t, 0)$ at time $t$ is described by the equation

$$
X(t, 0)=\int_{0}^{\infty} X(t, u) p(u) d u
$$

where $p(u)$ is the productivity of the equipment with age $u$, i.e. $p(u)$ is the production at age $u$ per capital unit. (In this model we only consider the part $X(t, u)$ of the produced capital that is reinvested into the production process.) 
We assume that the initial capital distribution $X(0, u)=\phi(u)$ is known. Then the solution $X(t, u)$ of $(1.16)$ is given by

$$
X(t, u)= \begin{cases}\phi(u-t) \cdot \exp \left(-\int_{0}^{t} m(s+u-t) d s\right) & ; 0 \leq t<u \\ X(t-u, 0) \cdot \exp \left(-\int_{0}^{u} m(s) d s\right) & ; t \geq u\end{cases}
$$

Substituting this in (1.17) we get the Volterra equation

$$
X(t, 0)=Y(t)+\int_{0}^{t} K(t-s) X(s, 0) d s
$$

where

$$
Y(t)=\int_{0}^{\infty} \phi(s) \exp \left(-\int_{0}^{t} m(s+r) d r\right) p(t+s) d s
$$

and

$$
K(t)=p(t) \exp \left(-\int_{0}^{t} m(s) d s\right)
$$

If the productivity function $p(u)$ is subject to random fluctuations we could model $p(u)$ by

$$
p(u)=p_{0}(u)+\epsilon W_{u}
$$

where $\epsilon>0$ and $W_{u}$ denotes white noise as before. This leads to a stochastic Volterra equation of the form (1.4) with $X_{t}=X(t, 0)$,

$$
b(t, s)=p_{0}(t-s) \exp \left(-\int_{0}^{t-s} m(r) d r\right) \quad ; \quad 0 \leq s \leq t
$$

$$
\sigma(t, s)=\epsilon \exp \left(-\int_{0}^{t-s} m(r) d r\right) \quad ; \quad 0 \leq s \leq t
$$

and

$$
\begin{aligned}
Y_{t} & =\int_{0}^{\infty} \phi(s) \exp \left(-\int_{0}^{t} m(s+r) d r\right) p_{0}(t+s) d s \\
& +\epsilon \int_{t}^{\infty} \phi(v-t) \exp \left(-\int_{0}^{t} m(v-t+r) d r\right) d B_{v}
\end{aligned}
$$

Note that $Y_{t}$ is not adapted in this case. 


\section{\$2. Some mathematical preliminaries}

Let $\left(\mathcal{S}^{\prime}, \mathcal{B}, \mu\right)$ denote the white noise probability space, i.e. $\mu$ is the probability measure on the Borel subsets $\mathcal{B}$ of the space $\mathcal{S}^{\prime}=\mathcal{S}^{\prime}(\mathbf{R})$ of tempered distributions on $\mathbf{R}$, with the property that

$$
\int_{S^{\prime}} e^{i<\omega, \phi>} d \mu(\omega)=e^{-\frac{1}{2}\|\phi\|^{2}}
$$

for all $\phi \in \mathcal{S}$, where $\|\phi\|^{2}=\int_{\mathbf{R}}|\phi|^{2} d x$ and $\langle\omega, \phi\rangle=\omega(\phi)$ is the action of $\omega \in \mathcal{S}^{\prime}$ (the dual of $\mathcal{S}$ ) on $\phi \in \mathcal{S}$. See [HKPS] for more information.

Recall that the white noise process $W$ is the map

$$
W: \mathcal{S} \times \mathcal{S}^{\prime} \rightarrow \mathbf{R}
$$

given by

$$
W(\phi, \omega)=W_{\phi}(\omega)=<\omega, \phi>; \phi \in \mathcal{S}, \omega \in \mathcal{S}^{\prime}
$$

i.e.

$$
W_{\phi}(\omega)=\int_{\mathbf{R}} \phi(t) d B_{t}
$$

where the right hand side denotes the Wiener-Ito integral with respect to Brownian motion $B_{t}$.

There is also a pointwise, singular version $W_{t}$ of white noise, which we describe below. Heuristically we may regard $W_{t}$ as the limit of $W_{\phi}$ as $\phi \rightarrow \delta_{t}$, the point mass at $t$. This limit exists in the space $(\mathcal{S})^{*}$ of Hida distributions. For definition and properties of $(\mathcal{S})^{*}$ see [HKPS]. An alternative description of $(\mathcal{S})^{*}$ can be given as follows (see [Z]): Let

$$
e_{n}(x)=\pi^{-\frac{1}{4}}((n-1) !)^{-\frac{1}{2}} e^{-\frac{2}{2}} h_{n-1}(\sqrt{2} x)
$$

be the Hermite function of order $n \geq 1$, where

$$
h_{m}(x)=(-1)^{m} e^{\frac{2}{2}} \frac{d^{m}}{d x^{m}} e^{-\frac{x^{2}}{2}}
$$

is the Hermite polynomial of order $m \geq 0$.

It is well known that $\left\{e_{n}\right\}_{n=1}^{\infty}$ forms an orthonormal base of $L^{2}=L^{2}(\mathrm{R})$. Moreover, $e_{n}$ is an eigenfunction for the operator

$$
A=-\frac{d^{2}}{d x^{2}}+x^{2}+1
$$

with eigenvalue $2 n$, i.e.

$$
A e_{n}=2 n e_{n}, n=1,2, \cdots
$$


Put $\theta_{j}(\omega)=\int_{\mathbf{R}} e_{j}(t) d B_{t}$ and define

$$
H_{\alpha}(\omega)=\prod_{j=1}^{m} h_{\alpha_{j}}\left(\theta_{j}\right)
$$

for all multi-indices $\alpha=\left(\alpha_{1}, \cdots, \alpha_{m}\right)$.

By the Wiener-Ito chaos theorem we have that each $f \in L^{2}(\mu)$ can be represented as a sum

$$
f(\omega)=\sum_{\alpha} c_{\alpha} H_{\alpha}(\omega)
$$

where

$$
\|f\|_{L^{2}(\mu)}^{2}=\sum_{\alpha} \alpha ! c_{\alpha}^{2}, \quad \text { and } \alpha !=\prod_{i=1}^{m} \alpha_{i} !
$$

We say that $f \in L^{2}(\mu)$ is a Hida test function, $f \in(\mathcal{S})$, if

$$
A_{f}(k):=\sup _{\alpha} c_{\alpha}^{2} \alpha !(2 \mathbf{N})^{\alpha k}<\infty \text { for all } k<\infty
$$

where

$$
(2 \mathrm{~N})^{\alpha}:=\prod_{j=1}^{m}(2 j)^{\alpha_{j}} \quad \text { if } \alpha=\left(\alpha_{1}, \cdots, \alpha_{m}\right) .
$$

The dual of $(\mathcal{S})$, denoted by $(\mathcal{S})^{*}$ (the space of Hida distributions) can be represented as the set of formal sums

$$
F=\sum_{\alpha} b_{\alpha} H_{\alpha}
$$

where

$$
\sup _{\alpha} b_{\alpha}^{2} \alpha !\left((2 \mathrm{~N})^{-\alpha}\right)^{q}<\infty \text { for some } q<\infty .
$$

The action of $F \in(\mathcal{S})^{*}$ (given by (2.11)) on the test function $f \in(\mathcal{S})$ (given by (2.7)) is

$$
<F, f>=\sum_{\alpha} \alpha ! b_{\alpha} c_{\alpha}
$$

In general we have

$$
(\mathcal{S}) \subset L^{p}(\mu) \subset(\mathcal{S})^{*} \text { for all } p \in(1, \infty)
$$

We can now define the pointwise white noise $W_{t}$ in $(\mathcal{S})^{*}$ by

$$
W_{t}(\omega)=\sum_{k=1}^{\infty} e_{k}(t) h\left(\theta_{k}\right)
$$


$\left(=\sum_{n=1}^{\infty} b_{n} H_{\epsilon_{n}}(\omega)\right.$ with $\epsilon_{n}=(0,0, \cdots, 1)$ with 1 on the n'th place and $\left.b_{n}=e_{n}(t)\right)$

Then

$$
\sup _{\alpha} b_{\alpha}^{2} \alpha !(2 \mathrm{~N})^{-\alpha q}=\sup _{n} e_{n}^{2}(t) \cdot 1 \cdot(2 n)^{-q}<\infty
$$

for $q>-\frac{1}{12}$, since $\left\|e_{n}\right\|_{\infty}=0\left(n^{-\frac{1}{12}}\right)$ as $n \rightarrow \infty$ (See [HP, Formula (21.3.3)]). So $W_{t} \in(\mathcal{S})^{*}$ as claimed.

In the following we will use the convention that $W_{t}$ means the pointwise white noise (in $\left.(\mathcal{S})^{*}\right)$ if $t \in \mathbf{R}$ while $W_{\psi}$ means the white noise defined by (2.3) if $\psi \in \mathcal{S}$. In spite of the ambiguouity of this notation we think it will be clear from the context what we mean.

If $F=\sum_{\alpha} a_{\alpha} H_{\alpha}$ and $G=\sum_{\beta} b_{\beta} H_{\beta}$ are two elements of $(\mathcal{S})^{*}$ we define their Wick product $F \diamond G$ as the element of $(\mathcal{S})^{*}$ given by

$$
F \diamond G=\sum_{\alpha, \beta} a_{\alpha} b_{\beta} H_{\alpha+\beta}=\sum_{\gamma}\left(\sum_{\alpha+\beta=\gamma} a_{\alpha} b_{\beta}\right) H_{\gamma}
$$

Using the characterization (2.9) one can prove that both $(\mathcal{S})^{*}$ and $(\mathcal{S})$ are closed under $\diamond$, i.e. $f, g \in(\mathcal{S})^{*} \Rightarrow f \diamond g \in(\mathcal{S})^{*}$ and similarly for $(\mathcal{S})$. (See the argument in [Z]).

There is an alternative to the representation (2.7): If $f \in L^{2}(\mu)$ there exist functions $f_{n} \in \hat{L}^{2}\left(\mathbf{R}^{n}\right)$ such that

$$
f(\omega)=\sum_{n=0}^{\infty} \int_{\mathbf{R}^{n}} f_{n}\left(t_{1}, \cdots, t_{n}\right) d B_{t}^{\otimes n}
$$

and

$$
\|f\|_{L^{2}(\mu)}^{2}=\sum_{n=0}^{\infty} n !\left\|f_{n}\right\|_{L^{2}\left(\mathrm{R}^{n}\right)}^{2}
$$

Here $\hat{L}^{2}\left(\mathbf{R}^{n}\right)$ denotes the space of symmetric $L^{2}$ - functions on $\mathbf{R}^{n}$ and $d B_{t}^{\otimes n}=d B_{t_{1}} d B_{t_{2}}$ $\cdots d B_{t_{n}}$ denotes the n'th iterated Ito integral. Using this representation the Wick product of two functions

$$
f=\sum_{n} \int f_{n} d B^{\otimes n} \quad \text { and } g=\sum_{m} \int g_{m} d B^{\otimes m}
$$

in $L^{2}(\mu)$ can be expressed as (when convergent)

$$
f \diamond g=\sum_{n, m} \int f_{n} \hat{\otimes} g_{m} d B^{\otimes(n+m)}
$$

where $\hat{\otimes}$ denotes the symmetrized tensor product.

The Wick product plays a crucial role in our solution of the stochastic Volterra equation. Since $L^{1}(\mu)$ is not contained in $(\mathcal{S})^{*}$, an extra definition is needed for that case (see [HLØUZ]):

Suppose there exist $X_{n}, Y_{n} \in L^{2}(\mu)$ such that $X_{n} \rightarrow X$ in $L^{1}(\mu), Y_{n} \rightarrow Y$ in $L^{1}(\mu), X_{n} \diamond Y_{n} \in$ $L^{1}(\mu)$ for all $n$ and $Z:=\lim X_{n} \diamond Y_{n}$ exists in $L^{1}(\mu)$. 
Then we define

$$
X \diamond Y=Z
$$

This definition does not depend on the specific choice of $\left\{X_{n}\right\},\left\{Y_{n}\right\}$. In fact, we have

$$
\mathcal{F}[X \diamond Y](\phi)=e^{\frac{1}{2}\|\phi\|^{2}} \mathcal{F}[X](\phi) \cdot \mathcal{F}[Y](\phi) \quad \forall \phi \in \mathcal{S}
$$

where

$$
\mathcal{F}[X](\phi)=\int_{\mathcal{S}^{\prime}} e^{i<\omega, \phi\rangle} X(\omega) d \mu(\omega) \quad, \phi \in \mathcal{S}
$$

is the Fourier transform of $X$.

(For a proof, see [HLØUZ, Lemma 9.2]). A survey of the properties of the Wick product is given in [GHL $\varnothing \mathrm{UZ}]$.

Now let us recall two important transforms on $(\mathcal{S})^{*}$ :

If $F \in(\mathcal{S})^{*}$ then the $\mathcal{S}$-transform of $F$ (first introduced in [KT]), $\mathcal{S} F$, is a map from $\mathcal{S}$ into $\mathbf{C}$ defined by

$$
\mathcal{S} F(\phi)=e^{-\frac{1}{2}\|\phi\|^{2}}<F, \exp <\cdot, \phi>>
$$

(It can be proved that the function $\omega \rightarrow \exp \langle\omega, \phi\rangle ; \omega \in \mathcal{S}^{\prime}$ belongs to $(\mathcal{S}$ ), so (2.21) is well-defined).

Note that if $F \in L^{2}(\mu)$ then

$$
\mathcal{S} F(\phi)=e^{-\frac{1}{2}\|\phi\|^{2}} \int_{\mathcal{S}^{\prime}} \exp (\langle\omega, \phi\rangle) F(\omega) d \mu(\omega)
$$

The Hermite transform (first introduced in [L $\varnothing \mathrm{U} 1]$ ) of $F, \mathcal{H} F$, is a map from the space $\mathbf{C}_{0}^{\mathbf{N}}$ of all finite sequences of complex numbers into $\mathbf{C}$ (the set of complex numbers) defined by

$$
\mathcal{H} F\left(z_{1}, z_{2}, \cdots\right):=\tilde{F}\left(z_{1}, z_{2}, \cdots\right)=\mathcal{S} F\left(z_{1} e_{1}+z_{2} e_{2}+\cdots\right) ;\left(z_{1}, z_{2}, \cdots\right) \in \mathrm{C}_{0}^{\mathrm{N}}
$$

Equivalently (see [L ØU1, Th. 5.7]) if $F \in(\mathcal{S})^{*}$ has the expansion

$$
F(\omega)=\sum_{\alpha} c_{\alpha} H_{\alpha}(\omega)
$$

then, using multi-index notation $z^{\alpha}=z_{1}^{\alpha_{1}} z_{2}^{\alpha_{2}} \cdots$ if $z=\left(z_{1}, z_{2}, \cdots\right)$ and $\alpha=\left(\alpha_{1}, \alpha_{2}, \cdots\right)$ we have

$$
\mathcal{H} F(z)=\sum_{\alpha} c_{\alpha} z^{\alpha} \quad ; z \in \mathbf{C}_{0}^{\mathbf{N}}
$$

It can be shown that the series converges and represents an analytic function of $z \in \mathbf{C}_{0}^{\mathrm{N}}$, for all $F \in(\mathcal{S})^{*}$ (see [HKPS]). The characterizations of the Wick product in terms of the $\mathcal{S}$ - and the $\mathcal{H}$ - transform are the following: 
If $F, G \in(\mathcal{S})^{*}$ then

$$
\mathcal{H}(F \diamond G)(z)=\mathcal{H} F(z) \cdot \mathcal{H} G(z) ; \quad z \in \mathbf{C}_{0}^{\mathrm{N}}
$$

and

$$
\mathcal{S}(F \diamond G)(\phi)=\mathcal{S F}(\phi) \cdot \mathcal{S} G(\phi) ; \quad \phi \in \mathcal{S}
$$

Finally we recall that there is an explicit inverse of the Hermite transform [L $\emptyset \mathrm{U} 1]$ :

Let $\lambda$ be the probability measure on $\mathbf{R}^{\mathbf{N}}$ defined by

$$
\int_{\mathbf{R}^{\mathbf{N}}} f\left(y_{1}, \cdots, y_{n}\right) d \lambda(y)=(2 \pi)^{-n / 2} \int_{\mathbf{R}^{n}} f(y) e^{-\frac{1}{2}|y|^{2}} d y
$$

if $f$ is a bounded measurable function of $y=\left(y_{1}, y_{2}, \cdots\right) \in \mathbf{R}^{\mathbf{N}}$ depending only on the first $n$ coordinates $y_{1}, \cdots, y_{n}$. Let

$$
X=\sum_{\alpha} c_{\alpha} H_{\alpha}(\omega) \text { be in } L^{2}(\mu)
$$

so that $X$ has the Hermite transform

$$
\tilde{X}(z)=\mathcal{H} X(z)=\sum_{\alpha} c_{\alpha} z^{\alpha}
$$

Then we recover $X$ from $\tilde{X}$ by

$$
\left.X(\omega):=\mathcal{H}^{-1} \tilde{X}:=\lim _{n, k \rightarrow \infty} \int_{\mathbb{R}^{\mathbb{N}}} \tilde{X}^{(n, k)}(\theta+i y) d \lambda(y) \quad \text { (limit in } L^{2}(\mu)\right)
$$

where $\theta+i y=\left(\theta_{1}+i y_{1}, \theta_{2}+i y_{2}, \cdots\right)$ with $\theta_{k}=\int e_{k} d B$ and

$$
\tilde{X}^{(n, k)}(z)=\sum_{\alpha \in J_{n, k}} c_{\alpha} z^{\alpha} ; J_{n, k}=\left\{\alpha ;|\alpha| \leq n \quad \text { and } \alpha_{j}=0 \quad \text { for } j>k\right\}
$$

is the doubly truncated series for $\tilde{X}$.

Moreover, we have the estimate [HLØUZ, Th. 4.2]:

$$
E\left[|X|^{p}\right] \leq \liminf _{n, k \rightarrow \infty} \iint\left|\tilde{X}^{(n, k)}(\xi+i \eta)\right|^{p} d \lambda(\xi) d \lambda(\eta)
$$

for all $p \in[1, \infty)$.

Before we finish this section, let us recall the main results in [PS] which will be used intensively in $\S 4$ :

DEFINITION 2.1. Let $F$ be a complex valued functional on $\mathcal{S}(\mathbf{R})$. We call $F$ a $U$ functional if the following conditions are satisfied: 
C.1 For all $\phi, \psi \in \mathcal{S}(\mathbf{R})$, the mapping $\lambda \rightarrow F(\psi+\lambda \phi), \lambda \in \mathbf{R}$, has an entire analytic extension, which will be denoted by $F(\psi+z \phi), z \in \mathbf{C}$

C.2 There exists a $p \in \mathbf{N}_{0}$ (the set of non-negative integers) and $C_{1}, C_{2}>0$ so that for all $z \in \mathbf{C}, \phi \in \mathcal{S}(\mathbf{R})$,

$$
|F(z \phi)| \leq C_{1} \exp \left(C_{2}|z|^{2}\|\phi\|_{2, p}^{2}\right)
$$

where $\|\phi\|_{2, p}=\left\|A^{p} \phi\right\|_{L^{2}(\mathrm{R})}$.

Then we have following theorems from [PS]:

THEOREM 2.1 [PS]. If $\Phi \in(\mathcal{S})^{*}$ then $\mathcal{S} \Phi$ is a $U$-functional. Conversely, if $F$ is a $U$-functional, then there is a unique $\Phi$ in $(\mathcal{S})^{*}$, so that $\mathcal{S} \Phi=F$.

THEOREM 2.2 [PS]. Assume that $F_{n}, n \in \mathrm{N}$ (the set of natural numbers), and $F$ are $U$ functionals and let $\Phi_{n}, n \in \mathbf{N}$, and $\Phi$, respectively, denote the associated Hida distributions in $(\mathcal{S})^{*}$. Then the following are equivalent:

(a) The sequence $\left(\Phi_{n}, n \in \mathrm{N}\right)$ converges strongly to $\Phi$

(b) The sequence $\left(F_{n}, n \in \mathbf{N}\right)$ converges pointwise to $F$ and for all large enough $n \in \mathbf{N}$, the estimate (2.31) holds for every $F_{n}$ uniformly in $n$. 


\section{§3. The functional process approach}

Functional processes were first introduced in [L ØU1] in a study of certain stochastic differential equations involving functionals of white noise. An extended multiparameter version was used in [HLØUZ]. Functional processes may be regarded as a generalization of distribution valued processes.

DEFINITION 3.1 Let $p>0$. A (one-parameter) $L^{p}$ functional process is a function

$$
X: \mathcal{S} \times \mathbf{R} \times \mathcal{S}^{\prime} \rightarrow \mathbf{R}
$$

such that

(i) the map $t \rightarrow X(\phi, t, \omega)$ is (Borel) measurable for each $\phi \in \mathcal{S}$ and a.a. $\omega \in \mathcal{S}^{\prime}$ and

(ii) the map $\omega \rightarrow X(\phi, t, \omega)$ is in $L^{p}(\mu)$ for each $\phi \in \mathcal{S}$ and each $t \in \mathbf{R}$

EXAMPLE 3.2 We may regard the white noise process as a functional process $W(\phi, t, \omega)$ by defining

$$
W(\phi, t, \omega)=W_{\phi_{t}}(\omega)=<\omega, \phi_{t}>
$$

where

$$
\phi_{t}(s)=\phi(s-t)
$$

is the $t$-shift of the test function $\phi$.

Note that if $F \in \mathcal{S}^{\prime}$ and $D$ denotes the differentiation operator we have

$$
<D F, \phi_{x}(\cdot)>=-<F, D \phi_{x}(\cdot)>=-<F, \frac{d}{d y} \phi(y-x)>=<F, \frac{d}{d x} \phi_{x}(y)>,
$$

so taking distributional derivatives of $F$ and applying the result to $\phi_{x}$ is the same as applying $F$ to the derivative of $\phi_{x}$ with respect to $x$. In view of this it is natural to interpret distributional differential equations with respect to $\phi$ involving functional processes $X(\phi, x, \omega)$ as ordinary differential equations in $x$ for each $\phi$.

The second observation which is relevant for the interpretation of (1.5) is the following:

LEMMA 3.3 Let $Y_{t}(\omega)$ be a stochastic process and let $\phi \in \mathcal{S}$ be such that

$$
Z_{t}:=(\phi * Y)_{t}
$$

satisfies condition (1.6). Then

$$
\int_{\mathbf{R}}(\phi * Y)_{t} \delta B_{t}=\int_{\mathbf{R}} Y_{t} \diamond W_{\phi_{t}} d t
$$

where $*$ denotes convolution, i.e.

$$
(\phi * Y)_{t}(\omega)=\int_{\mathbf{R}} \phi(t-s) Y_{s}(\omega) d s
$$


Proof. By Corollary 3.4 in [LØU2] we have

$$
\int_{\mathbf{R}} Z_{t} \delta B_{t}=\int_{\mathbf{R}} Z_{t} \diamond W_{t} d t
$$

for all processes $Z_{t}$ satisfying (1.6), where the right hand side is regarded as an element in $(\mathcal{S})^{*}$.

Applying this to $Z_{t}=(\phi * Y)_{t}$ we get

$$
\begin{aligned}
& \int_{\mathbf{R}}(\phi * Y)_{t} \delta B_{t}=\int_{\mathbf{R}}\left(\int_{\mathbf{R}} \phi(t-s) Y_{s}(\omega) d s\right) \diamond W_{t} d t \\
& =\int_{\mathbf{R}} Y_{s}(\omega) \diamond\left(\int_{\mathbf{R}} \phi(t-s) W_{t} d t\right) d s \\
& =\int_{\mathbf{R}} Y_{s}(\omega) \diamond W_{\phi_{s}} d s, \quad \text { as required. }
\end{aligned}
$$

In view of Lemma 2.1 and (3.3) the following interpretation of (1.5) is natural:

We say that a functional process $X(\phi, t, \omega)=X_{t}^{\phi}$ is a solution of (1.5) if, for all $\phi \in \mathcal{S}$,

$$
X_{t}^{\phi}=Y_{t}^{\phi}+\int_{0}^{t} b(t, s) X_{s}^{\phi} d s+\int_{0}^{t} \sigma(t, s) X_{s}^{\phi} \diamond W_{\phi s} d s \quad t \geq 0
$$

where $Y_{t}^{\phi}=Y(\phi, t, \omega)$ is a given functional process.

LEMMA 3.4 Let $b(t, s)$ and $\sigma(t, s)$ be two bounded deterministic functions satisfying

$$
b(t, s)=\sigma(t, s)=0 \quad \text { if } \quad 0 \leq t<s
$$

Fix $\phi \in \mathcal{S}$ and define, for $(t, s) \in[0, \infty) \times[0, \infty)$,

$$
K_{1}(t, s):=K(t, s):=b(t, s)+\sigma(t, s) W_{\phi_{s}}(\omega)
$$

and inductively

$$
K_{n+1}(t, s)=\int_{0}^{t} K_{n}(t, u) \diamond K(u, s) d u\left(=\int_{s}^{t} K_{n}(t, u) \diamond K(u, s) d u\right) \quad ; n \geq 1 .
$$

Then for all $(t, s)$

$$
\left\|K_{n}(t, s)\right\|_{L^{2}(\mu)} \leq \frac{M^{n}(1+\|\phi\|)^{n}}{\sqrt{n !}} \quad ; \quad n=1,2, \cdots
$$

and therefore the series

$$
H(t, s, \omega):=\sum_{n=1}^{\infty} K_{n}(t, s, \omega)
$$


converges in $L^{2}(\mu)$ uniformly for $(t, s) \in[0, \infty) \times[0, \infty)$.

Proof. First note that, if we put

$$
\gamma(t, s)=\gamma(t, s, \omega)=\sigma(t, s) W_{\phi_{s}}
$$

then

$$
\begin{aligned}
K_{2}(t, s) & =\int_{s}^{t} K(t, u) \diamond K(u, s) d u \\
& =\int_{s}^{t}(b(t, u)+\gamma(t, u)) \diamond(b(u, s)+\gamma(u, s)) d u
\end{aligned}
$$

and

$$
\begin{aligned}
K_{3}(t, s) & =\int_{s}^{t} K_{2}(t, v) \diamond K(v, s) d v \\
& =\int_{s \leq v \leq u \leq t} \int_{0}[(b(t, u)+\gamma(t, u)) \diamond(b(u, v)+\gamma(u, v)) \diamond(b(v, s)+\gamma(v, s))] d u d v
\end{aligned}
$$

So by induction

$$
K_{n}(t, s)=\int_{s \leq u_{n-1} \leq \ldots \leq u_{1} \leq t} \ldots \int_{0 \leq k \leq n-1}\left[\prod_{0}^{\circ}\left(b\left(u_{k}, u_{k+1}\right)+\gamma\left(u_{k}, u_{k+1}\right)\right)\right] d u_{1} \cdots d u_{n-1},
$$

where $u_{0}=t$ and $u_{n}=s$ and $\stackrel{\ominus}{\prod}$ indicates that the Wick product is used.

Now

$$
\prod_{0 \leq k \leq n-1}^{\circ}\left(b\left(u_{k}, u_{k+1}\right)+\gamma\left(u_{k}, u_{k+1}\right)\right)=\sum_{\alpha, \beta} b_{\alpha}(u) \gamma_{\beta}(u)
$$

the sum being taken over all partitions $\{\alpha, \beta\}$ of $\{0,1, \cdots, n-1\}$ (i.e. $\alpha \cap \beta=\emptyset$ and $\alpha \cup \beta=\{0,1, \cdots, n-1\})$ and we have used the notation

$$
b_{\alpha}(u)=b\left(u_{\alpha_{1}}, u_{\alpha_{1}+1}\right) \cdots b\left(u_{\alpha_{j}}, u_{\alpha_{j}+1}\right) \quad \text { if } \quad \alpha=\left\{\alpha_{1}, \cdots, \alpha_{j}\right\}
$$

and

$$
\gamma_{\beta}(u)=\gamma\left(u_{\beta_{1}}, u_{\beta_{1}+1}\right) \diamond \cdots \diamond \gamma\left(u_{\beta_{k}}, u_{\beta_{k+1}}\right) \quad \text { if } \quad \beta=\left\{\beta_{1}, \cdots, \beta_{k}\right\}
$$

Since

$$
E\left[\left|W_{\phi_{u_{1}}} \diamond \cdots \diamond W_{\phi_{u_{k}}}\right|^{2}\right]^{1 / 2}=\sqrt{k !}\|\phi\|^{k}
$$

we obtain from (3.12) that (with $|\beta|=$ the cardinality of $\beta$ )

$$
\left\|K_{n}(t, s)\right\|_{L^{2}(\mu)} \leq \int_{s \leq u_{n-1} \leq \cdots \leq u_{1} \leq t} \ldots \int_{\alpha, \beta}\left|b_{\alpha}(u)\right| \cdot\left|\sigma_{\beta}(u)\right| \sqrt{|\beta| !}\|\phi\|^{|\beta|} d u_{1} \cdots d u_{n-1}
$$


Choose $M<\infty$ such that

$$
|b(t, s)| \leq M \text { and }|\sigma(t, s)| \leq M \text { for all } t, s .
$$

Then from (3.16) we get (putting $|\beta|=k$ )

$$
\begin{aligned}
\left\|K_{n}(t, s)\right\|_{L^{2}(\mu)} & \leq M^{n} \cdot \frac{1}{n !} \cdot \sum_{k=0}^{n}\left(\begin{array}{l}
n \\
k
\end{array}\right) \sqrt{k !}\|\phi\|^{k} \\
& \leq \frac{M^{n}}{\sqrt{n !}}(1+\|\phi\|)^{n} .
\end{aligned}
$$

LEMMA 3.5. Let $K, K_{n}$ be as in Lemma 3.4. Then

$$
K_{n}(t, s)=\int_{s}^{t} K_{n-j}(t, u) \diamond K_{j}(u, s) d u \quad \text { for } \quad j=1,2, \cdots, n-1 .
$$

Proof. We proceed by induction. By definition (3.17) holds for $j=1$ for all $n$. Suppose (3.17) holds for $n=n_{0}$ and all $j \leq n_{0}-1$ and also for $n=n_{0}+1$ if $j=j_{0} \leq n_{0}-1$. Then

$$
\begin{aligned}
& \int_{s}^{t} K_{n_{0}+1-\left(j_{0}+1\right)}(t, u) \diamond K_{j_{0}+1}(u, s) d u \\
& =\int_{s}^{t} K_{n_{0}-j_{0}}(t, u) \diamond\left(\int_{s}^{u} K_{j_{0}}(u, v) \diamond K(v, s) d v\right) d u \\
& =\int_{s}^{t} K(v, s) \diamond\left(\int_{v}^{t} K_{n_{0}-j_{0}}(t, u) \diamond K_{j_{0}}(u, v) d u\right) d v \\
& =\int_{s}^{t} K(v, s) \diamond K_{n_{0}}(t, v) d v=K_{n_{0}+1}(t, s) .
\end{aligned}
$$

LEMMA 3.6 Let $K(t, s)$ and $H(t, s)$ be as in Lemma 3.4. Then

$$
H(t, s)-K(t, s)=\int_{s}^{t} K(t, u) \diamond H(u, s) d u
$$

Proof. By (3.9) and (3.7) we have

$$
\begin{aligned}
& \int_{s}^{t} K(t, u) \diamond H(u, s) d u=\lim _{N \rightarrow \infty} \int_{s}^{t}\left(\sum_{n=1}^{N} K(t, u) \diamond K_{n}(u, s)\right) d u \\
& =\lim _{N \rightarrow \infty} \sum_{n=1}^{N} K_{n+1}(t, s)=H(t, s)-K(t, s) .
\end{aligned}
$$


We are now ready for the first main result of this section:

THEOREM 3.7 Let $b(t, s)$ and $\sigma(t, s)$ be bounded deterministic functions satisfying

$$
b(t, s)=\sigma(t, s)=0 \quad \text { if } \quad 0 \leq t<s
$$

Let $Y_{t}=Y(\phi, t, \omega)$ be an $L^{1}$ functional process (not necessarily adapted) such that

$$
\sum_{m=1}^{\infty}\left\|K_{m}(s, u) \diamond Y_{u} \diamond K_{n}(t, s)\right\|_{L^{2}(\mu)} \leq C<\infty \quad \text { for all } t, s, u, n
$$

(with $C$ independent of $t, s, u$ and $n$ ) where $K_{n}$ is defined by (3.7). Define

$$
X_{t}:=X(\phi, t, \omega):=Y_{t}+\int_{0}^{t} H(t, s) \diamond Y_{s} d s
$$

Then

$$
X_{s} \diamond K_{n}(t, s) \in L^{1}(d s \times d \mu) \text { for all } t, n
$$

and $X_{t}$ is the unique $L^{1}$ functional process which satisfies (3.20) and solves the ((1.11) interpretation of the) stochastic Volterra equation

$$
X_{t}=Y_{t}+\int_{0}^{t} b(t, s) X_{s} d s+\int_{0}^{t} \sigma(t, s) X_{s} \delta B_{s}
$$

Proof. We first verify that $X_{t}$ given by (3.19) satisfies (3.20): From (3.18) we have

$$
\begin{aligned}
& \left(\int_{0}^{s} H(s, u) \diamond Y_{u} d u\right) \diamond K_{n}(t, s)=\left(\sum_{m=1}^{\infty} \int_{0}^{s} K_{m}(s, u) \diamond Y_{u} d u\right) \diamond K_{n}(t, s) \\
& =\sum_{m=1}^{\infty} \int_{0}^{s} K_{m}(s, u) \diamond Y_{u} \diamond K_{n}(t, s) d u
\end{aligned}
$$

which converges absolutely in $L^{2}(d s \times d \mu)$

Next we verify that if $X_{t}$ is defined by (3.19) then $X_{t}$ satisfies (1.11), i.e.

$$
X_{t}=Y_{t}+\int_{0}^{t} X_{s} \diamond K(t, s) d s
$$


Substituting for $X$ and using Lemma 3.5 we get

$$
\begin{aligned}
& \int_{0}^{t} X_{s} \diamond K(t, s) d s=\int_{0}^{t} Y_{s} \diamond K(t, s) d s+\int_{0}^{t}\left(\int_{0}^{s} H(s, u) \diamond Y_{u} d u\right) \diamond K(t, s) d s \\
& =\int_{0}^{t} Y_{s} \diamond K(t, s) d s+\int_{0}^{t}\left(Y_{u} \diamond \int_{u}^{t} K(t, s) \diamond H(s, u) d s\right) d u \\
& =\int_{0}^{t} Y_{s} \diamond K(t, s) d s+\int_{0}^{t} Y_{u} \diamond(H(t, u)-K(t, u)) d u \\
& =\int_{0}^{t} Y_{u} \diamond H(t, u) d u=X_{t}-Y_{t},
\end{aligned}
$$

which proves that $X_{t}$ defined by (3.19) satisfies (1.5).

It remains to prove uniqueness:

Suppose $X_{t}^{(1)}, X_{t}^{(2)}$ are two $L^{1}$ functional processes such that for all $t$

$$
X_{s}^{(i)} \diamond K_{n}(t, s) \in L^{1}(d \mu \times d s) \text { for } i=1,2 .
$$

Then since both processes satisfying (3.21) we get by subtraction that

$$
Z_{s}:=X_{s}^{(1)}-X_{s}^{(2)}
$$

satisfies the equation

$$
Z_{t}=\int_{0}^{t} K(t, s) \diamond Z_{s} d s
$$

This gives

$$
\begin{aligned}
Z_{t} & =\int_{0}^{t} K(t, s) \diamond\left(\int_{0}^{s} K(s, u) \diamond Z_{u} d u\right) d s \\
& =\int_{0}^{t} K_{2}(t, u) \diamond Z_{u} d u
\end{aligned}
$$

Proceeding by induction we see that

$$
Z_{t}=\int_{0}^{t} K_{n}(t, u) \diamond Z_{u} d u \text { for all } n
$$

Applying the $\mathcal{F}$-transform on both sides we get

$$
\mathcal{F} Z_{t}(\phi)=e^{\frac{1}{2}\|\phi\|^{2}} \int_{0}^{t}\left(\mathcal{F} K_{n}(t, u)\right)(\phi)\left(\mathcal{F} Z_{u}\right)(\phi) d u \quad \phi \in \mathcal{S} .
$$


Since $K_{n}(t, u) \rightarrow 0$ in $L^{2}(\mu)$ as $n \rightarrow \infty$, uniformly in $(t, u)$, we see that

$$
\left(\mathcal{F} K_{n}(t, u)\right)(\phi) \rightarrow 0 \text { as } n \rightarrow \infty \text {, uniformly in }(t, u)
$$

and we conclude from (3.22) that $\mathcal{F} Z_{t}(\phi)=0$ for all $\phi$. Therefore $Z_{t}=0$, which proves uniqueness.

The following is an important special case of Theorem 3.7:

THEOREM 3.8 Let $b(t, s), \sigma(t, s)$ be as in Theorem 3.7. Suppose $Y_{t}=Y(\phi, t, \omega)$ satisfies (3.18) and in addition that $Y_{t}$ is independent of $t$, i.e.

$$
Y_{t}=Y_{0} \text { for all } t
$$

Then the unique $L^{1}$ functional process $X_{t}$ which satisfies (3.20) and solves the Volterra equation

$$
X_{t}=Y_{0}+\int_{0}^{t} b(t, s) X_{s} d s+\int_{0}^{t} \sigma(t, s) X_{s} \delta B_{s}
$$

is given by

$$
X_{t}:=X(\phi, t, \omega)=Y_{0} \diamond\left(1+\int_{0}^{t} H(t, s) d s\right)
$$

REMARK. Note that the unique solution $x_{t}(\omega)$ of the non-anticipating Volterra equation

$$
x_{t}^{(a)}=a+\int_{0}^{t} b(t, s) x_{s}^{(a)} d s+\int_{0}^{t} \sigma(t, s) x_{s}^{(a)} d B_{s} \quad(a \text { constant })
$$

is (by Theorem 3.8)

$$
x_{t}^{(a)}=a\left(1+\int_{0}^{t} H(t, s) d s\right)
$$

The connection between the solution $x_{t}^{(a)}$ of the non-anticipating equation (3.26) and the solution $X_{t}$ of the anticipating equation (3.24) is therefore

$$
X_{t}=Y_{0} \diamond x_{t}^{(1)}
$$

In particular, note that if $Y_{0}$ is anticipating then $X_{t}$ does not coincide with $x_{t}^{(a)}$ with $a=Y_{0}$ ! Condition (3.18) may be difficult to use in specific cases. In order to get a more tractable condition we establish the following result of independent interest:

LEMMA 3.9 Let $b, \sigma$ be as in Theorem 3.7 and let $x_{t}=x_{t}^{(1)}$ be the (non-anticipating) solution of (3.26). Let $\mathcal{H} x_{t}=\tilde{x}_{t}$ be the Hermite transform of $x_{t}$. Then (with $\lambda$ as in $\S 2$ )

$$
\iint\left|\tilde{x}_{t}(x+i y)\right|^{p} d \lambda(x) d \lambda(y)<\infty \text { for all } p<\infty
$$


Proof. Taking $\mathcal{H}$-transforms of (3.26) we get

$$
\tilde{x}_{t}(z)=1+\int_{0}^{t} b(t, s) \tilde{x}_{s}(z) d s+\int_{0}^{t} \sigma(t, s) \tilde{x}_{s}(z) \tilde{W}_{\phi_{s}}(z) d s
$$

with $\tilde{W}_{\phi_{s}}(z)=\sum_{n}\left(\phi_{s}(\cdot), e_{n}\right) z_{n} ; z=\left(z_{1}, \cdots, z_{2}, \cdots\right) \in \mathbf{C}_{0}^{\mathbf{N}}$, where $\left(\psi, e_{n}\right)=\int_{\mathbf{R}} \psi e_{n} d x$ denotes the inner product in $L^{2}(\mathbf{R})$.

If we choose $M$ such that

$$
|b(t, s)| \leq M \text { and }|\sigma(t, s)| \leq M \text { for all } t, s
$$

then

$$
\left|\tilde{x}_{t}(z)\right| \leq 1+M \int_{0}^{t}\left|\tilde{x}_{s}\right| d s+M \cdot \int_{0}^{t}\left|\tilde{x}_{s}\right| \cdot\left|\tilde{W}_{\phi_{s}}\right| d s
$$

By the Gronwall inequality (see e.g. [EK, Appendix 5])

$$
\begin{aligned}
\left|\tilde{x}_{t}(z)\right| & \leq \exp \left(M t+M \int_{0}^{t}\left|\tilde{W}_{\phi_{s}}\right| d s\right) \\
& \leq \exp \left(M t+M \int_{0}^{t}\left(\sum_{n}\left|\left(\phi_{s}, e_{n}\right)\right|\left|x_{n}\right|+\sum_{n}\left|\left(\phi_{s}, e_{n}\right)\right| \cdot\left|y_{n}\right|\right) d s\right) \\
& \leq \exp (M t) \cdot \exp \left(M \sum_{n}\left(\int_{0}^{t}\left|\left(\phi_{s}, e_{n}\right)\right| d s\right) \cdot\left|x_{n}\right|+\sum_{n}\left(\int_{0}^{t}\left|\left(\phi_{s}, e_{n}\right)\right| d s\right) \cdot\left|y_{n}\right|\right)
\end{aligned}
$$

Therefore, if we put $a_{n}=M p \cdot \int_{0}^{t}\left|\left(\phi_{s}, e_{n}\right)\right| d s$,

$$
\begin{aligned}
& \iint\left|\tilde{x}_{t}(z)\right|^{p} d \lambda(x) d \lambda(y) \leq \exp (p M t) \cdot\left[\int \exp \left(\sum_{n} a_{n}\left|x_{n}\right|\right) d \lambda(x)\right]^{2} \\
& =\exp (p M t) \prod_{n}\left[\int_{\mathbf{R}} \exp \left(a_{n}\left|x_{n}\right|\right) e^{-\frac{\left|x_{n}\right|^{2}}{2}} \frac{d x_{n}}{\sqrt{2 \pi}}\right]^{2}
\end{aligned}
$$

If $a>0$ we have

$$
\begin{aligned}
& \int_{\mathbf{R}} \exp \left(a|t|-\frac{1}{2} t^{2}\right) \frac{d t}{\sqrt{2 \pi}} \\
& =\exp \left(\frac{1}{2} a^{2}\right)\left[\int_{-\infty}^{0} \exp \left(-\frac{1}{2}(t+a)^{2}\right) \frac{d t}{\sqrt{2 \pi}}+\int_{0}^{\infty} \exp \left(-\frac{1}{2}(t-a)^{2}\right) \frac{d t}{\sqrt{2 \pi}}\right] \\
& =\exp \left(\frac{1}{2} a^{2}\right)\left[\int_{-\infty}^{a}+\int_{-a}^{\infty} \exp \left(-\frac{1}{2} y^{2}\right) \frac{d y}{\sqrt{2 \pi}}\right] \\
& =\exp \left(\frac{1}{2} a^{2}\right)\left[1+2 \int_{0}^{a} \exp \left(-\frac{1}{2} y^{2}\right) \frac{d y}{\sqrt{2 \pi}}\right] \leq \exp \left(\frac{1}{2} a^{2}\right)[1+2 a] .
\end{aligned}
$$


Next consider (with the operator $A$ is in (2.5))

$$
\begin{aligned}
\sum_{n=1}^{\infty} a_{n} & =p M \cdot \sum_{n=1}^{\infty} \int_{0}^{t}\left|\left(\phi_{s}, e_{n}\right)\right| d s=p M \int_{0}^{t}\left(\sum_{n=1}^{\infty}\left|\left(\phi_{s}, e_{n}\right)\right|\right) d s \\
& =p M \int_{0}^{t} \sum_{n=1}^{\infty}\left|\left(A^{-2} e_{n}, A^{2} \phi_{s}\right)\right| d s \\
& \leq p M \int_{0}^{t} \sum_{n=1}^{\infty}\left\|A^{-2} e_{n}\right\|_{L^{2}}\left\|A^{2} \phi_{s}\right\|_{L^{2}} d s \\
& \leq p M \int_{0}^{t} \sum_{n=1}^{\infty}(2 n)^{-2}\left\|A^{2} \phi_{s}\right\|_{L^{2}} d s \\
& =p M t\left\|A^{2} \phi\right\|_{L^{2}} \cdot \sum_{n=1}^{\infty}(2 n)^{-2}<\infty
\end{aligned}
$$

since $\phi \in \mathcal{S}$ and $A^{2} \phi_{s}(x)=\left(A^{2} \phi\right)_{s}(x)$.

Combining (3.31) with (3.32) and (3.33) we get

$$
\begin{aligned}
& \iint\left|\tilde{x}_{t}(z)\right|^{p} d \lambda(x) d \lambda(y) \leq \exp (p M t) \prod_{n=1}^{\infty} \exp \left(a_{n}^{2}\right)\left[1+2 a_{n}\right]^{2} \\
& =\exp (p M t) \cdot \exp \left(\sum_{n=1}^{\infty} a_{n}^{2}+2 \ln \left(1+2 a_{n}\right)\right) \\
& \leq \exp (p M t) \cdot \exp \left(\sum_{n=1}^{\infty} a_{n}^{2}+4 a_{n}\right)<\infty .
\end{aligned}
$$

If we apply Lemma 3.9 in Theorem 3.8 we get the following:

THEOREM 3.10. Suppose $b(t, s), \sigma(t, s)$ are as in Theorem 3.7 and suppose $Y_{0}=$ $Y_{0}(\phi, \omega)$ satisfies

$$
\tilde{Y}_{0} \in L^{1+\epsilon}(\lambda \times \lambda) \text { for some } \epsilon=\epsilon(\phi)>0, \forall \phi \in \mathcal{S} .
$$

Then

$$
X_{t}=Y_{0} \diamond x_{t}^{(1)}=Y_{0} \diamond\left(1+\int_{0}^{t} H(t, s) d s\right)
$$

is the unique $L^{1}$ functional process which satisfies (3.20) and solves the Volterra equation

$$
X_{t}=Y_{0}+\int_{0}^{t} b(t, s) X_{s} d s+\int_{0}^{t} \sigma(t, s) X_{s} \delta B_{s}
$$




\section{§4. The generalized white noise functional approach}

In this section, we consider the following equation (4.1) in the Hida distribution setting,

$$
X_{t}=Y_{t}+\int_{0}^{t} b(t, s) X_{s} d s+\int_{0}^{t} \sigma(t, s) X_{s} \diamond W_{s} d s
$$

where $Y_{t}$ is regarded as a process in $(\mathcal{S})^{*}$.

Throughout this section, we assume that $b(t, s)$ and $\sigma(t, s)$ are bounded deterministic functions satisfying

$$
b(t, s)=\sigma(t, s)=0 \quad \text { if } \quad 0 \leq t<s .
$$

Now let us state our first result:

THEOREM 4.1. Assume there exist constants $c_{1}, c_{2}>0, p \in \mathrm{N}_{0}$, independent of $t$, such that the estimate (2.31) holds for $F=\mathcal{S} Y_{t}$ for all $t$. Then the equation (4.1) has a unique solution $X_{t}$ in $(\mathcal{S})^{*}$, which is given by

$$
X_{t}=Y_{t}+\int_{0}^{t} H(t, s) \diamond Y_{s} d s
$$

where

$$
\begin{gathered}
H(t, s)=\sum_{\nu=1}^{\infty} K_{\nu}(t, s) \\
K_{1}(t, s)=b(t, s)+\sigma(t, s) W_{s} \\
K_{\nu+1}(t, s)=\int_{0}^{t} K_{1}(t, u) \diamond K_{\nu}(u, s) d u ; \nu=1,2, \cdots
\end{gathered}
$$

The series (4.4) converges strongly in $(\mathcal{S})^{*}$.

Proof. It is enough to construct the solution on any fixed interval $[0, T]$. We divide the proof into several steps.

LEMMA 4.2. $K_{\nu}(t, s)$ is a well-defined generalized functional for all $\nu, s, t$.

Proof. By proposition 2.6 in [PS] and Theorem 3.1 in [Po], it suffices to prove that $\mathcal{S} K_{\nu}(t, s)$ can be bounded in the sense of (2.31) uniformly in $t, s$. We see this by induction:

Since $b(t, s)$ and $\sigma(t, s)$ are bounded, it is clear that there exist constants $c_{1}>0, p_{1} \in \mathbf{N}_{0}$, independent of $t, s$, such that for $\phi \in \mathcal{S}(\mathbf{R})$

$$
\left|\mathcal{S} K_{1}(t, s)(z \phi)\right| \leq c_{1} \exp \left(c_{1}|z|\|\phi\|_{2, p_{1}}\right)
$$


Suppose there are constants $c_{\nu}, p_{\nu} \in \mathbf{N}_{0}$ such that

$$
\left|\mathcal{S} K_{\nu}(t, s)(z \phi)\right| \leq c_{\nu} \exp \left(c_{\nu}|z|\|\phi\|_{2, p_{\nu}}\right)
$$

for $\phi \in \mathcal{S}, t, s \leq T$.

By (4.5) we have

$$
\begin{aligned}
& \left|\mathcal{S} K_{\nu+1}(t, s)(z \phi)\right| \leq \int_{0}^{t}\left|\mathcal{S} K_{1}(t, u)(z \phi)\right| \cdot\left|\mathcal{S} K_{\nu}(u, s)(z \phi)\right| d u \\
& \leq \int_{0}^{t} c_{1} \exp \left(c_{1}|z|\|\phi\|_{2, p_{1}}\right) \cdot c_{\nu} \exp \left(c_{\nu}|z|\|\phi\|_{2, p_{\nu}}\right) d u \\
& \leq c_{\nu+1} \exp \left(c_{\nu+1}|z|\|\phi\|_{2, p_{\nu+1}}\right) \quad \text { for } \quad \phi \in \mathcal{S}(\mathrm{R}), t, s \leq T .
\end{aligned}
$$

where $c_{\nu+1}=\max \left(T c_{1} c_{\nu}, c_{1}+c_{\nu}\right), p_{\nu+1}=p_{1} \vee p_{\nu}$. This completes the proof of Lemma 4.2.

LEMMA 4.3. The series $H(t, s)=\sum_{\nu=1}^{\infty} K_{\nu}(t, s)$ converges strongly in $(\mathcal{S})^{*}$.

We first show that $\hat{H}(t, s)(\phi):=\sum_{\nu=1}^{\infty} \mathcal{S} K_{\nu}(t, s)(\phi)$ is a $U$-functional. By the definition, we need to verify the following two claims :

Claim 1: $\hat{H}(t, s)(\psi+z \phi)$ is an entire function of $z \in \mathbf{C}$ for any $\psi, \phi \in \mathcal{S}(\mathbf{R})$.

Proof of Claim 1. Since $\mathcal{S} K_{\nu}(t, s)(\psi+z \phi)$ is analytic, it is sufficient to show that $\sum_{\nu=1}^{\infty} \mathcal{S} K_{\nu}(t, s)(\psi+z \phi)$ converges uniformly on compact sets in $\mathbf{C}$.

In fact, for any $M>0$, put $\hat{K}(t, s, z)=b(t, s)+\sigma(t, s)(\psi(s)+z \phi(s))$ and

$$
\begin{aligned}
\lambda(t, s) & =\sup _{|z| \leq M}|\hat{K}(t, s, z)| \\
A^{2}(t) & =\int_{0}^{T} \lambda^{2}(t, u) d u, B^{2}(s)=\int_{0}^{T} \lambda^{2}(u, s) d u .
\end{aligned}
$$

Then

$$
\begin{aligned}
& \sup _{|z| \leq M}\left|S K_{2}(t, s)(\psi+z \phi)\right|^{2} \\
& \leq \sup _{|z| \leq M}\left|\int_{s}^{t} \hat{K}(t, u, z) \hat{K}(u, s, z) d u\right|^{2} \\
& \leq \sup _{|z| \leq M}\left(\int_{s}^{t}|\hat{K}(t, u, z)|^{2} d u\right)\left(\int_{s}^{t}|\hat{K}(u, s, z)|^{2} d u\right) \\
& \leq \int_{0}^{T} \lambda^{2}(t, u) d u \int_{0}^{T} \lambda^{2}(u, s) d u \\
& =A^{2}(t) B^{2}(s)
\end{aligned}
$$


Similarly

$$
\begin{aligned}
& \sup _{|z| \leq M}\left|\mathcal{S} K_{3}(t, s)(\psi+z \phi)\right|^{2} \\
& \leq \sup _{|z| \leq M}\left|\int_{s}^{t} \hat{K}(t, u, z) \mathcal{S} K_{2}(u, s)(\psi+z \phi) d u\right|^{2} \\
& \leq A^{2}(t) B^{2}(t) \int_{s}^{t} A^{2}(u) d u
\end{aligned}
$$

Inductively we have

$$
\begin{aligned}
& \sup _{|z| \leq M}\left|\mathcal{S} K_{\nu+2}(t, s)(\psi+z \phi)\right|^{2} \\
& \leq A^{2}(t) B^{2}(s) \frac{1}{\nu !}\left(\int_{0}^{T} A^{2}(u) d u\right)^{\nu}
\end{aligned}
$$

This implies that $\sum_{\nu=1}^{\infty} \mathcal{S} K_{\nu}(t, s)(\psi+z \phi)$ converges uniformly on $|z| \leq M$. Hence the claim follows.

Claim 2: There exist $c_{1}, c_{2}>0$ and $p \in \mathbf{N}_{0}$ such that

$$
|\hat{H}(t, s)(z \phi)| \leq c_{1} \exp \left(c_{2}|z|^{2}\|\phi\|_{2, p}^{2}\right)
$$

for $\phi \in \mathcal{S}(\mathbf{R}), t, s \leq T$.

Proof of Claim 2. Set

$$
\begin{aligned}
& \lambda(t, s, z)=|b(t, s)+\sigma(t, s) z \phi(s)| \\
& A^{2}(t, z)=\int_{0}^{T} \lambda^{2}(t, u, z) d u \\
& B^{2}(s, z)=\int_{0}^{T} \lambda^{2}(u, s, z) d u
\end{aligned}
$$

As the proof of (4.10), we get that

$$
\left|\mathcal{S} K_{\nu+2}(t, s)(z \phi)\right| \leq A(t, z) B(s, z) \frac{\left(\int_{0}^{T} A^{2}(u, z) d u\right)^{\nu / 2}}{\sqrt{\nu !}}
$$

Thus

$$
\begin{aligned}
|\hat{H}(t, s)(z \phi)| & \leq \lambda(t, s, z)+\sum_{\nu=0}^{\infty}\left|\mathcal{S} K_{\nu+2}(t, s)(z \phi)\right| \\
& \leq \lambda(t, s, z)+A(t, z) B(s, z) \sum_{\nu=0}^{\infty} \frac{1}{\sqrt{\nu !}}\left(\int_{0}^{T} A^{2}(u, z) d u\right)^{\nu / 2} \\
& \leq \lambda(t, s, z)+A(t, z) B(s, z) \sqrt{2} \exp \left(2 \int_{0}^{T} A^{2}(u, z) d u\right)
\end{aligned}
$$


On the other hand, it is easy to see that there exist constants $c_{3}, c_{4}>0$ and $p_{1} \in \mathbf{N}_{0}$ such that

$$
\begin{aligned}
\int_{0}^{T} A^{2}(u, z) d u & \leq c_{3}+c_{4} \int_{0}^{T} \phi(u)^{2} d u \\
|A(t, z)| & \leq c_{3}+c_{4}|z|\left(\int_{0}^{T} \phi(u)^{2} d u\right)^{\frac{1}{2}} \\
|B(s, z)| & \leq c_{3}+c_{4}|z|\|\phi\|_{2, p_{1}} \\
|\lambda(t, s, z)| & \leq c_{3}+c_{4}|z|\|\phi\|_{2, p_{1}}
\end{aligned}
$$

for all $\phi \in \mathcal{S}(\mathbf{R}), t, s \leq T$.

Combining (4.14) with (4.13), we have (4.11).

Now let us complete the proof of Lemma 4.3. Let $H(t, s) \in(\mathcal{S})^{*}$ with $\mathcal{S}(H(t, s))(\phi)=$ $\hat{H}(t, s)(\phi)$. Put $\Phi_{n}=\sum_{\nu=1}^{n} K_{\nu}(t, s)$. Then we see that

$$
\mathcal{S} \Phi_{n}(\phi) \rightarrow \mathcal{S}(H(t, s))(\phi) \quad \text { for } \quad \phi \in \mathcal{S}(\mathbf{R})
$$

and $\left|\Phi_{n}(z \phi)\right|$ is uniformly controlled by (4.11). Applying Theorem 2.2, we obtain Lemma 4.3.

LEMMA 4.4. $X_{t}$ (given by (4.3)) is well-defined and satisfies (4.1).

Proof. By the assumption, there is some $p_{1} \in \mathbf{N}_{0}$ so that

$$
\left|\mathcal{S} Y_{t}(z \phi)\right| \leq c \exp \left(c|z|^{2}\|\phi\|_{2, p_{1}}^{2}\right)
$$

for $\phi \in \mathcal{S}(\mathbf{R})$, all $t>0$.

Combining with (4.11), we obtain that for some $p \in \mathrm{N}_{0}, c_{1}>0$

$$
\left|\mathcal{S} Y_{s}(z \phi) \mathcal{S} H(t, s)(z \phi)\right| \leq c_{1} \exp \left(c_{1}|z|\|\phi\|_{2, p}^{2}\right)
$$

Here the constant $c_{1}$ is independent of $s, t, \phi, z$. Again from proposition 2.6 in [PS] and Theorem 3.1 in [Po] we conclude that $X_{t}=Y_{t}+\int_{0}^{t} H(t, s) \diamond Y_{s}$ is well-defined, with $\mathcal{S}$ transform:

$$
\mathcal{S} X_{t}(\phi)=\mathcal{S} Y_{t}(\phi)+\int_{0}^{t} \hat{H}(t, s)(\phi) \mathcal{S} Y_{s}(\phi) d s .
$$

Likewise,

$$
Z_{t}:=Y_{t}+\int_{0}^{t} b(t, s) X_{s} d s+\int_{0}^{t} \sigma(t, s) X_{s} \diamond W_{s} d s
$$


is also a well defined process in $(\mathcal{S})^{*}$. From our construction and Theorem 1.5 in $[\mathrm{T}]$, we know that $\mathcal{S} X_{t}=\mathcal{S} Z_{t}$. This means that $X_{t}$ satisfies (4.1). The uniqueness follows from the uniqueness of the solution of the following deterministic equation:

$$
\hat{Z}_{t}(\phi)=\mathcal{S} Y_{t}(\phi)+\int_{0}^{t} b(t, s) \hat{Z}_{s}(\phi) d s+\int_{0}^{t} \sigma(t, s) \hat{Z}_{s}(\phi) \phi(s) d s .
$$

This completes the proof of Theorem 4.1.

\section{REMARKS.}

(1): In particular, if $Y_{t}=Y_{0} \in(\mathcal{S})^{*}$ in Theorem 4.1, then $X_{t}=Y_{0} \diamond x_{t}^{(1)}$ where as before $x_{t}^{(1)}$ is the solution of the equation

$$
x_{t}^{(1)}=1+\int_{0}^{t} b(t, s) x_{s}^{(1)} d s+\int_{0}^{t} \sigma(t, s) x_{s}^{(1)} d B_{s}
$$

This formula is a natural extension of the non-anticipating case. See Theorem 3.8 and the Remark there.

(2): There is a close connection between the Hida distribution solution $X_{t} \in(\mathcal{S})^{*}$ of equation (4.1) and the functional process solution $X_{t}^{\phi}$ of equation (1.11) found in $\S 3$. To see this put

$$
\phi(x)= \begin{cases}\operatorname{cexp}\left(\frac{1}{|x|^{2}-1}\right) & |x| \leq 1 \\ 0 & |x|>1\end{cases}
$$

with $c$ chosen such that $\int_{\mathbf{R}} \phi(x) d x=1$.

Set $\phi^{(\epsilon)}(x)=\frac{1}{\epsilon} \phi\left(\frac{x}{\epsilon}\right)$ for $\epsilon>0$. Then for any $\psi \in \mathcal{S}(\mathbf{R}), \phi^{(\epsilon)} * \psi \rightarrow \psi$. Let $X_{t}^{(\epsilon)}$ be the solution found in Section 3 of equation (1.11) with $\phi:=\phi^{(\epsilon)}$. If $Y_{t}^{(\epsilon)}:=Y_{t}^{\phi^{(\epsilon)}}$ is good enough then $\mathcal{S} X_{t}^{(\epsilon)}(\psi)$ satisfies

$$
\begin{aligned}
\mathcal{S} X_{t}^{(\epsilon)}(\psi)=\mathcal{S} Y_{t}^{(\epsilon)}(\psi) & +\int_{0}^{t} b(t, s) \mathcal{S} X_{s}^{(\epsilon)}(\psi) d s \\
& +\int_{0}^{t} \sigma(t, s) \mathcal{S} X_{s}^{(\epsilon)}(\psi) \phi^{(\epsilon)} * \psi(s) d s \quad \text { for all } \epsilon>0
\end{aligned}
$$

Thus if $Y_{t}^{(\epsilon)} \rightarrow Y_{t}$ in $(\mathcal{S})^{*}$, then by Theorem 2.2 one can see that $X_{t}^{(\epsilon)} \rightarrow X_{t}$ in $(\mathcal{S})^{*}$, where $X_{t}$ is the solution of equation (4.1).

In the rest of this section, we treat a special case

$$
\begin{aligned}
& \sigma(t, s)=f(t) \sigma(s), \quad b(t, s)=f(t) b(s) \\
& Y_{t} \equiv Y_{0}=\sum_{n=0}^{\infty} \int_{\mathbb{R}^{n}} F_{n}\left(t_{1} \cdots t_{n}\right) d B_{t}^{\otimes n} \in L^{2}(\mu)
\end{aligned}
$$


We will prove that in this situation the solution of equation (4.1) is actually in $L^{2}(\mu)$ :

\section{THEOREM 4.5}

(I) Assume $\delta \leq f \leq \frac{1}{\delta}$ ( $\delta$ is a positive constant), $f^{\prime} \in L_{\text {loc }}^{2}\left(\mathbf{R}_{+}\right),\|A \sigma f\|_{L^{2}(\mathbf{R})}<+\infty$. Let $Y_{t} \equiv Y_{0}$ as in (4.18) and assume that $\sum_{n=0}^{\infty} n !\left\|A^{\otimes n} F_{n}\right\|_{L^{2}\left(\mathbf{R}^{n}\right)}^{2}<+\infty$. Then the solution $X_{t}$ of (4.1) is in $L^{2}(\mu)$ and

$$
\begin{aligned}
X_{t} & =-\int_{0}^{t} \frac{f(t) f^{\prime}(s)}{f^{2}(s)}\left(Y_{0} \diamond \exp \left(X_{t}^{(0)}-X_{s}^{(0)}-\frac{1}{2}\left(<X^{(0)}>_{t}-<X^{(0)}\right\rangle_{s}\right)\right) d s \\
& +\frac{f(t)}{f(0)}\left(Y_{0} \diamond \exp \left(X_{t}^{(0)}-\frac{1}{2}<X^{(0)}>_{t}\right)\right)
\end{aligned}
$$

where $X_{t}^{(0)}=\int_{0}^{t} \sigma(s) f(s) d B_{s}+\int_{0}^{t} b(s) f(s) d s$.

(II) Assume $f^{\prime} \in L_{\text {loc }}^{4}\left(\mathbf{R}_{+}\right), \delta \leq f \leq \frac{1}{\delta}$. Let $Y_{t} \equiv Y_{0} \in L^{2}(\mu)$ and assume that

$$
\iint\left|\mathcal{H} Y_{0}(\xi+i \eta)\right|^{4} d \lambda(\xi) d \lambda(\eta)<+\infty
$$

where $d \lambda$ is as in $\S 2$.

Then $X_{t} \in L^{2}(\mu)$ and (4.19) holds.

Proof. (I): In this case the solution $X_{t} \in(\mathcal{S})^{*}$ found in Theorem 4.1 satisfies

$$
\mathcal{S} X_{t}(\phi)=\mathcal{S} Y_{0}(\phi)+\int_{0}^{t} f(t) b(s) \mathcal{S} X_{s}(\phi) d s+\int_{0}^{t} f(t) \sigma(s) \phi(s) \mathcal{S} X_{s}(\phi) d s
$$

Set $\bar{g}_{t}(\phi)=\frac{s X_{t}(\phi)}{f(t)}$, then

$$
\bar{g}_{t}(\phi)=\frac{\mathcal{S} Y_{0}(\phi)}{f(t)}+\int_{0}^{t} b(s) f(s) \bar{g}_{s}(\phi) d s+\int_{0}^{t} \sigma(s) f(s) \bar{g}_{s}(\phi) \phi(s) d s
$$

Therefore we have

$$
\begin{aligned}
\mathcal{S} X_{t}(\phi) & =-\int_{0}^{t} \frac{f(t) f^{\prime}(s)}{f^{2}(s)} \mathcal{S} Y_{0}(\phi) \exp \left(\int_{s}^{t} \sigma(u) f(u) \phi(u) d u+\int_{s}^{t} b(u) f(u) d u\right) \\
& +\frac{f(t)}{f(0)} \mathcal{S} Y_{0}(\phi) \exp \left(\int_{0}^{t} \sigma(s) f(s) \phi(s) d s+\int_{0}^{t} b(s) f(s) d s\right)
\end{aligned}
$$

This gives the formula (4.19).

We denote by $X_{t}^{(1)}$ and $X_{t}^{(2)}$ the two parts of the R.H.S in (4.19). Then

$$
\mathcal{S} X_{t}^{(1)}(\phi)=-\int_{0}^{t} \frac{f(t) f^{\prime}(s)}{f^{2}(s)} \mathcal{S} Y_{0}(\phi) \exp \left(\int_{s}^{t} \sigma(u) f(u) \phi(u) d u+\int_{s}^{t} b(u) f(u) d u\right) d s
$$


Now suppose $X_{t}^{(1)} \in(\mathcal{S})^{*}$ has the formal expansion

$$
X_{t}^{(1)}=\sum_{n=0}^{\infty} \int_{\mathbb{R}^{n}} G_{n, t}\left(s_{1}, \cdots, s_{n}\right) d B_{s}^{\otimes n} \quad(\text { See }[\mathrm{PS}])
$$

Since $\mathcal{S} X_{t}^{(1)}(\phi)=\sum_{n=0}^{\infty}<G_{n, t}, \phi^{\otimes n}>$ (see [PS]), (4.24) indicates that

$$
G_{n, t}=-\int_{0}^{t} \frac{f(t) f^{\prime}(s)}{f^{2}(s)} \exp \left(\int_{s}^{t} b(u) f(u) d u\right) \sum_{m=0}^{n} F_{n-m} \hat{\otimes} \frac{\left(\bar{\sigma}_{s, t}\right)^{\otimes m}}{m !} d s
$$

where $\bar{\sigma}_{s, t}(u)=\sigma(u) f(u) 1_{[s, t]}(u)$. Therefore,

$$
\begin{aligned}
\left\|G_{n, t}\right\|_{L^{2}\left(\mathrm{R}^{n}\right)}^{2} & \leq\left(\int_{0}^{t} \frac{f^{2}(t) f^{\prime}(s)^{2}}{f^{4}(s)} \exp \left(2 \int_{s}^{t} b(u) f(u) d u\right) d s\right) \int_{0}^{t}\left\|\sum_{m=0}^{n} F_{n-m} \hat{\otimes} \frac{\left(\bar{\sigma}_{s, t}\right)^{\otimes m}}{m !}\right\|_{L^{2}\left(\mathrm{R}^{n}\right)}^{2} d s \\
& \leq C_{t} \int_{0}^{t}(n+1) \sum_{m=0}^{n}\left\|F_{n-m}\right\|_{L^{2}\left(\mathrm{R}^{n-m}\right)}^{2} \frac{\left(\left\|\bar{\sigma}_{s, t}\right\|_{L^{2}(\mathrm{R})}^{2}\right)^{m}}{(m !)^{2}} d s \\
& \leq C_{t}(n+1) t \sum_{m=0}^{n}\left\|F_{n-m}\right\|_{L^{2}\left(\mathrm{R}^{n-m}\right)}^{2} \frac{\left(\|\bar{\sigma}\|_{L^{2}(\mathrm{R})}^{2}\right)^{m}}{(m !)^{2}}
\end{aligned}
$$

where $\bar{\sigma}(u)=\sigma(u) f(u)$ and $C_{t}$ is a constant.

Thus

$$
\begin{aligned}
& \sum_{n=0}^{\infty} n !\left\|G_{n, t}\right\|_{L^{2}\left(\mathrm{R}^{n}\right)}^{2} \leq t C_{t} \sum_{n=0}^{\infty}(n+1) \sum_{m=0}^{n}\left(\begin{array}{c}
n \\
m
\end{array}\right)(n-m) !\left\|F_{n-m}\right\|_{L^{2}\left(\mathrm{R}^{n-m}\right)}^{2} \frac{\left(\|\bar{\sigma}\|_{L^{2}(\mathrm{R})}^{2}\right)^{m}}{m !} . \\
& \leq t C_{t} \sum_{n=0}^{\infty} \sum_{m=0}^{n}(n+1)\left(\begin{array}{c}
n \\
m
\end{array}\right) 2^{-2 n}(n-m) !\left\|A^{\otimes n-m} F_{n-m}\right\|_{L^{2}}^{2} \frac{\left(\|A \bar{\sigma}\|_{L^{2}}^{2}\right)^{m}}{m !} \\
& \leq t C_{t} \sum_{n=0}^{\infty} \sum_{m=0}^{n}(n-m) !\left\|A^{\otimes n-m} F_{n-m}\right\|_{L^{2}}^{2} \frac{\left(\|A \bar{\sigma}\|_{L^{2}}^{2}\right)^{m}}{m !} \\
& \leq t C_{t}\left(\sum_{n=0}^{\infty} n !\left\|A^{\otimes n} F_{n}\right\|_{L^{2}}^{2}\right) \exp \left(\|A \bar{\sigma}\|_{L^{2}}^{2}\right)<+\infty
\end{aligned}
$$

This shows that $X_{t}^{(1)} \in L^{2}(\mu)$. Similarly, we have $X_{t}^{(2)} \in L^{2}(\mu)$.

(II): By the estimate (2.30), it is sufficient to prove

$$
\iint\left|\mathcal{S} X_{t}\left(\left(\xi_{1}+i \eta_{1}\right) e_{1}+\cdots+\left(\xi_{n}+i \eta_{n}\right) e_{n}+\cdots\right)\right|^{2} d \lambda(\xi) d \lambda(\eta)<+\infty
$$


In fact, from (4.23) we know that

$$
\begin{aligned}
& \lim _{N \rightarrow \infty} \iint\left|\mathcal{S} X_{t}\left(\left(\xi_{1}+i \eta_{1}\right) e_{1}+\cdots+\left(\xi_{N}+i \eta_{N}\right) e_{N}\right)\right|^{2} d \lambda(\xi) d \lambda(\eta) \\
& \leq C_{0}\left(\iint\left|\mathcal{H} Y_{0}(\xi+i \eta)\right|^{4} d \lambda(\xi) d \lambda(\eta)\right) \times \\
& \lim _{N \rightarrow \infty} \iint\left|\exp \left(\int_{s}^{t} \sigma(u) f(u)\left(\left(\xi_{1}+i \eta_{1}\right) e_{1}+\cdots+\left(\xi_{N}+i \eta_{N}\right) e_{N}\right) d u\right)\right|^{4} d \lambda(\xi) d \lambda(\eta) \\
& \leq C_{1}\left(\lim _{N \rightarrow \infty} \int \exp \left(4 \sum_{i=1}^{n} \xi_{i} \int_{0}^{t} \sigma(u) f(u) e_{i}(u) d u\right) d \lambda(\xi)\right) . \\
& \leq C_{1} \exp \left(8 \int_{0}^{t} \sigma^{2}(u) f^{2}(u) d u\right)<+\infty .
\end{aligned}
$$

where $C_{0}$ and $C_{1}$ are appropriate constants. This ends the proof of (II) and the proof of Theorem 4.5 is complete.

\section{Acknowledgements}

This work is supported by the Norwegian Research Council for Science and the Humanities (NAVF), grant 410.91/001, and by VISTA, a research cooperation between the Norwegian Academy of Science and Letters and Den Norske Stats Oljeselskap A.S. (Statoil).

We are grateful to Kjell Arne Brekke for useful discussions.

\section{REFERENCES}

[BM] M.A. Berger and V.J. Mizel: An extension of the stochastic integral. The Annals of Probability 10 (1982), 435-450.

[EK] S. Ethier and T.G. Kurtz: Markov Processes. Characterization and Convergence. J. Wiley \& Sons 1986.

[GHLØUZ] G. Gjessing, H. Holden, T. Lindstrøm, B. Øksendal, J. Ubøe and T.-S. Zhang: The Wick product. Preprint University of Oslo 1992.

[GLS] G. Gripenberg, S.-O. Londen and O. Staffans: Volterra Integral and Functional Equations. Cambridge University Press 1990.

[HKPS] T. Hida, H.-H. Kuo, J. Potthoff and L. Streit: White Noise. (Forthcoming book).

[HLØUZ] H. Holden, T. Lindstrøm, B. Øksendal, J. Ubøe and T.S. Zhang: Stochastic boundary value problems: A white noise functional approach. Preprint University of Oslo 1991.

[HP] E. Hille and R.S. Phillips: Functional Analysis and Semigroups. Amer. Math. Soc. Colloq. Publ. 31 (1957). 
[KT] I. Kubo and S. Takenaka: Calculus on Gaussian white noise I. Proc. Japan Acad. 56 (1980), 376-380.

[LøU1] T. Lindstrøm, B. Øksendal and J. Ubøe: Stochastic differential equations involving positive noise: In M. Barlow and N. Bingham (editors): Stochastic Analysis. Cambridge Univ. Press 1991, 261-303.

[LøU2] T. Lindstrøm, B. Øksendal and J. Ubøe: Wick multiplication and Ito-Skorohod stochastic differential equations. To appear in S. Albeverio et al (editors): Ideas and Methods in Mathematics Analysis. Cambridge Univ. Press 1992.

[LøU3] T. Lindstrøm, B. Øksendal and J. Ubøe: Stochastic modelling of fluid flow in porous media. Preprint, University of Oslo 1991.

[NZ] D. Nualart and M. Zakai: Generalized stochastic integrals and the Malliavin calculus. Probab. Th. Rel. Fields 73 (1986), 255-280.

[O] S. Ogawa: On the stochastic integral equation of Fredholm type. Studies in Mathematics and its Applications 18 (1986); Patterns and Waves-Qualitative Analysis of Nonlinear Differential Equations, pp. 597-605.

[Pa] E. Pardoux: Applications of anticipating stochastic calculus to stochastic differential equations. In H. Korezlioglu and A.S. Ustunel (eds): Stochastic Analysis and Related Topics II. Springer LNM 1444 (1990), 63-105.

[Po] J. Potthoff: White noise methods for stochastic partial differential equations. Manuscript 1991.

[PP] E. Pardoux and P. Protter: Stochastic Volterra equations with anticipating coefficients. The Annals of Probability 18 (1990), 1635-1655.

[PS] J. Potthoff and L. Streit: A characterization of Hida distributions. J. Funct. Anal. 101 (1991), 212-229.

[T] F.G. Tricomi: Integral Equations. Dover 1985.

[Z] T.-S. Zhang: Characterizations of white noise test functions and Hida distributions. Preprint University of Oslo 1991 (To appear in Stochastics). 
